\title{
Face-Transitive Polyhedra with Rectangular Faces and Icosahedral Symmetry
}

\author{
H. S. M. Coxeter ${ }^{1}$ and Branko Grünbaum ${ }^{2}$ \\ ${ }^{1}$ Department of Mathematics, University of Toronto, \\ Toronto, Ontario, Canada M5S 1A1 \\ 2 Department of Mathematics, University of Washington, \\ Box 354350, Seattle, WA $98195-4350$, USA \\ grunbaum@math.washington.edu
}

\begin{abstract}
We describe three hexacontahedra in which the faces are rectangles, all equivalent under symmetries of the icosahedral group and having all edges in the mirror planes of the symmetry group. Under the restriction that adjacent faces are not coplanar, these are the only possible polyhedra of this kind.
\end{abstract}

\section{Introduction}

In a recent paper [2] we described two face-transitive polyhedra $P_{1}$ and $P_{2}$, with nonsquare rectangular faces and octahedral symmetry, each edge of which lies in a mirror plane of the polyhedron. We indicated then that more complicated polyhedra with similar properties will be presented later. The aim of this note is to describe three polyhedra with icosahedral symmetry, in which all faces are non-square rectangles equivalent under symmetries of the polyhedron, and in which each edge is in a plane of mirror symmetry. Moreover, we restrict attention to polyhedra in which there are no coplanar faces. As in [2], the polyhedra described here can lead to other isohedra with rectangular faces if subdivision of faces or other modifications are allowed; these are briefly discussed in Section 6. However, the three polyhedra described below and denoted by $P \# 1, P \# 2$ and $P \# 3$ are the only ones that possess all the properties just mentioned.

\section{The Polyhedra}

Each of the three polyhedra has sixty faces and is thus a hexacontahedron; each has self-intersections of the kind familiar from the Kepler-Poinsot regular polyhedra. As 


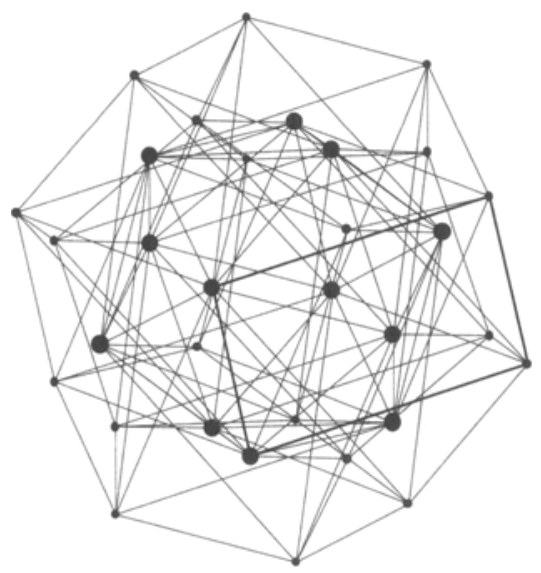

(a)

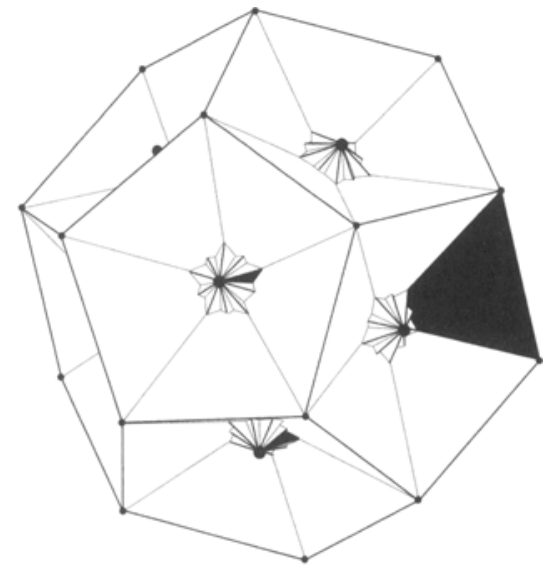

(b)

Fig. 1. (a) A skeletal view of the rectangle-faced hexacontahedron $P \# 1=\{5,3\} \&\{3,5\}$. The edges of one face are boldly drawn. Its vertices are those of a great stellated triacontahedron, with vertices of different orbits shown by solid dots of two sizes. (b) A perspective view of a model of the hexacontahedron $P \# 1=$ $\{5,3\} \&\{3,5\}$. The three visible parts of one face are shown in black. It is the face emphasized in the skeletal view.

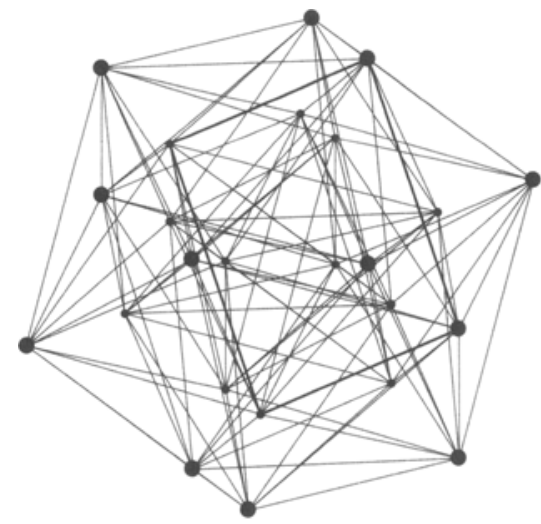

(a)

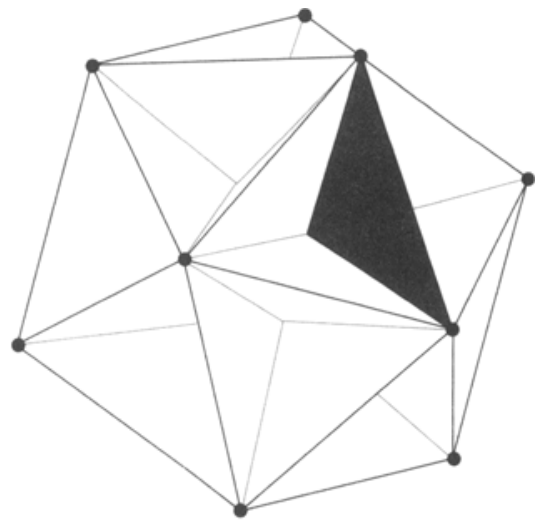

(b)

Fig. 2. (a) A skeletal view of the rectangle-faced hexacontahedron $P \# 2=\left\{5, \frac{5}{2}\right\} \&\left\{\frac{5}{2}, 5\right\}$. The edges of one face are boldly drawn. Its vertices are those of a small stellated triacontahedron, with vertices of different orbits shown by solid dots of two sizes. (b) A perspective view of a model of the hexacontahedron $P \# 2=$ $\left\{5, \frac{5}{2}\right\} \&\left\{\frac{5}{2}, 5\right\}$. The only visible part of one face is shown in black. It is the face emphasized in the skeletal view. 


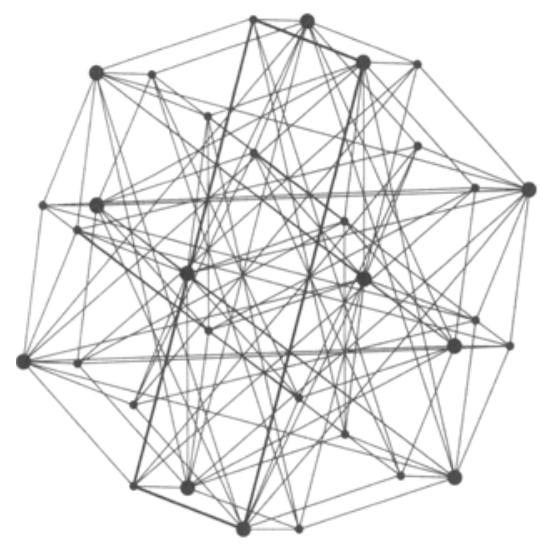

(a)

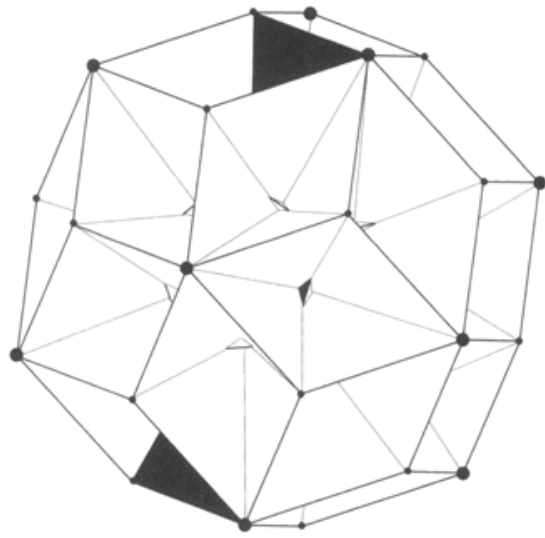

(b)

Fig. 3. (a) A skeletal view of the rectangle-faced hexacontahedron $P \# 3=\left\{3, \frac{5}{2}\right\} \&\left\{\frac{5}{2}, 3\right\}$. The edges of one face are boldly drawn. Its vertices are those of a rhombic triacontahedron, with vertices of different orbits shown by solid dots of two sizes. (b) A perspective view of a model of the hexacontahedron $P \# 3=\left\{3, \frac{5}{2}\right\} \&\left\{\frac{5}{2}, 3\right\}$. The three visible parts of one face are shown in black. It is the face emphasized in the skeletal view.

may be seen from Figs. 1-3, the polyhedra are quite complicated, and the diagrams are of little help in visualizing them or investigating their properties. Hence in Section 3 we describe three methods of construction of these polyhedra, which facilitate their study in different ways.

Each of the three hexacontahedra is shown in skeletal view as well as in perspective view of a cardboard model. For greater clarity, vertices are shown by solid dots. For each polyhedron, the vertices form two orbits under isometric symmetries; the two types are indicated by dots of different sizes. For additional intelligibility, in the skeletal view the edges of one face are heavily drawn, while in the views of the models the parts of one face that are visible from the outside are shown in black. Additional information about these three hexacontahedra is given in Sections 4 and 5.

\section{Three Constructions for the Hexacontahedra}

Among the various ways to describe the three rectangle-faced hexacontahedra, we present here three methods for their construction.

3.1. For the first method, we observe that the six regular polyhedra with icosahedral symmetry form three reciprocal pairs:

$$
\{5,3\} \text { and }\{3,5\}, \quad\left\{5, \frac{5}{2}\right\} \text { and }\left\{\frac{5}{2}, 5\right\}, \quad\left\{\frac{5}{2}, 3\right\} \text { and }\left\{3, \frac{5}{2}\right\} \text {. }
$$

When the polyhedra in such a pair are actually reciprocal with respect to a concentric sphere, each pair of opposite edges of one polyhedron is parallel to a pair of edges of the other that lie in the midplane of the first pair of edges. If, moreover, the reciprocating radius is adjusted so that both the polyhedra have the same edge length, then quadruplets 


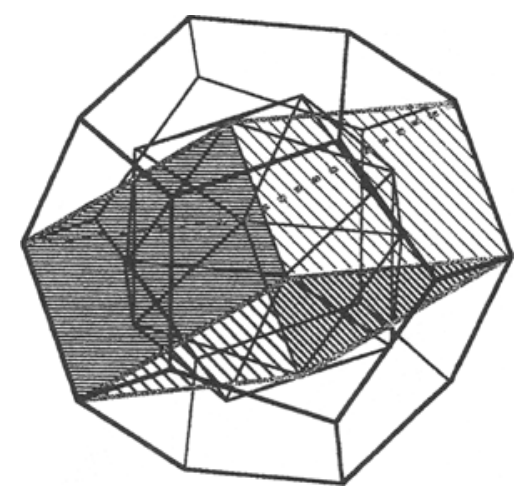

Fig. 4. The first construction of $P \# 1=\{5,3\} \&\{3,5\}$, based on reciprocal polyhedra $\{5,3\}$ and $\{3,5\}$. In order to avoid crowding the diagram, here and in Figs. 5 and 6, only one "zone" of four rectangles is indicated. Each edge of $\{5,3\}$ participates in one such a zone, and so does each edge of $\{3,5\}$. The other edges of the rectangles determine twelve zones of five faces each.

of edges as just described determine a zone or "tunnel", the walls of which are four congruent rectangles. This zone is bounded by two quadruples of edges, each forming a rhomb, and each edge has one vertex in each of the polyhedra. Since each polyhedron has thirty edges, there are fifteen such zones, each formed by four rectangles: sixty rectangles altogether. These sixty congruent rectangles are the faces of an isohedron, and the three pairs of reciprocals yield three such hexacontahedra which we designate

$$
P \# 1=\{5,3\} \&\{3,5\}, \quad P \# 2=\left\{5, \frac{5}{2}\right\} \&\left\{\frac{5}{2}, 5\right\}, \quad \text { and } \quad P \# 3=\left\{\frac{5}{2}, 3\right\} \&\left\{3, \frac{5}{2}\right\} .
$$

This construction is illustrated in Figures 4-6, in each of which only one zone of four rectangles is shown.

3.2. For the second method we start with the net of Möbius triangles for the icosahedral group [1, p. 111], see Fig. 7; as is well known, this is the partition of the sphere

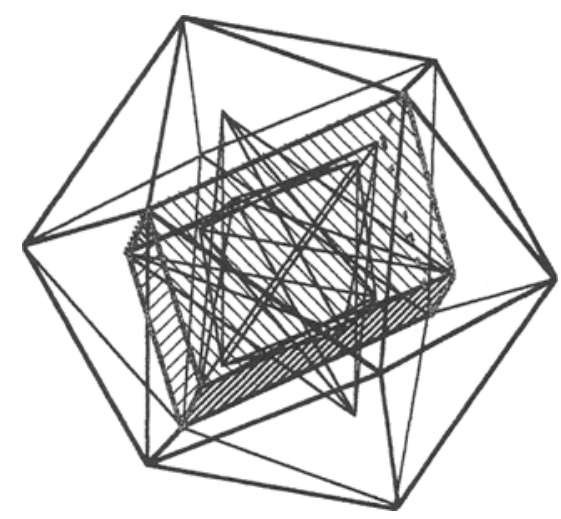

Fig. 5. The first construction of $P \# 2=\left\{5, \frac{5}{2}\right\} \&\left\{\frac{5}{2}, 5\right\}$, based on reciprocal polyhedra $\left\{5, \frac{5}{2}\right\}$ and $\left\{\frac{5}{2}, 5\right\}$. 


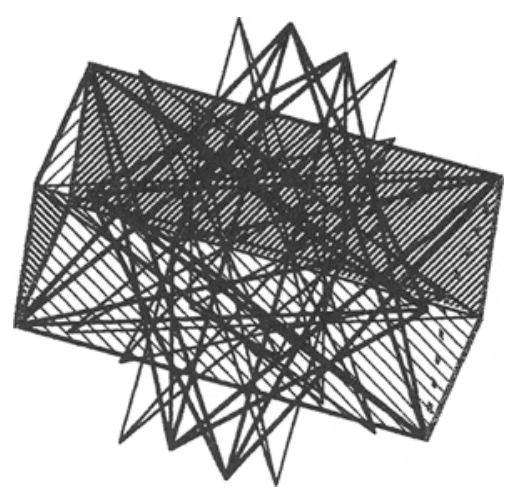

Fig. 6. The first construction of $P \# 3=\left\{3, \frac{5}{2}\right\} \&\left\{\frac{5}{2}, 3\right\}$, based on reciprocal polyhedra $\left\{3, \frac{5}{2}\right\}$ and $\left\{\frac{5}{2}, 3\right\}$.

by the planes of mirror symmetry of the icosahedral group. We search for (spherical) quadrangles $A B C D$, the sides of which are arcs of the great circles forming the net, and which themselves have mirror symmetry in one of the midlines, say the one bisecting $A B$ and $C D$, see Fig. 8. On the rays from the center $O$ of the sphere through the points $C, D$ we locate points $C^{*}, D^{*}$ in such a way that $O C^{*}=O D^{*}$ and $A B=C^{*} D^{*}$. Then the planar quadrangle $A B C^{*} D^{*}$ is a rectangle, all sides of which are in mirrors of the icosahedral group. The action of the group on this rectangle creates a polyhedron of the type we are interested in.

This method of construction can be used to find the complete list of rectangle-faced polyhedra; it shows, in particular, that the three polyhedra described here are the only ones satisfying the criteria specified in the Introduction. More details about this use of Möbius nets appear in Section 6 below.

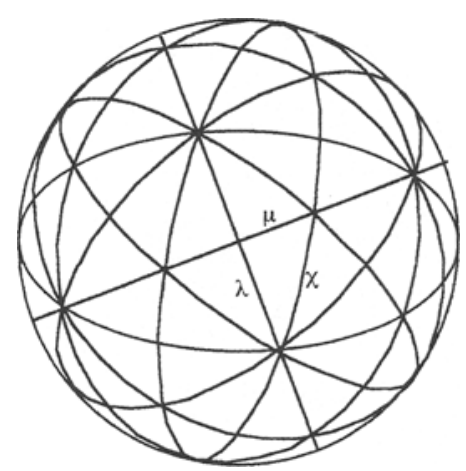

Fig. 7. The icosahedral Möbius net on the sphere. The sizes of the sides of each triangle of the Möbius net are $\lambda=\frac{1}{2} \arctan 2=31.7174744^{\circ}, \mu=\frac{1}{2} \arcsin \frac{2}{3}=20.90505745^{\circ}$, and $\chi=\pi / 2-\lambda-\mu=37.37736814^{\circ}$. 


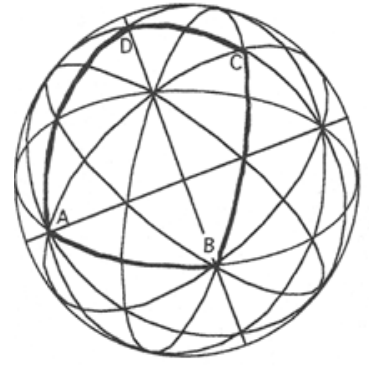

(a)

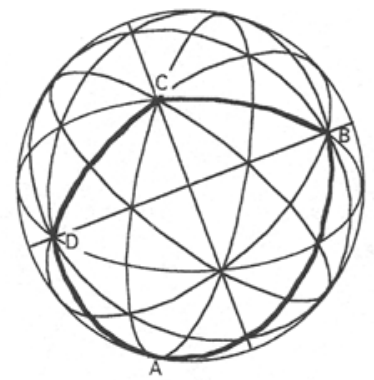

(b)

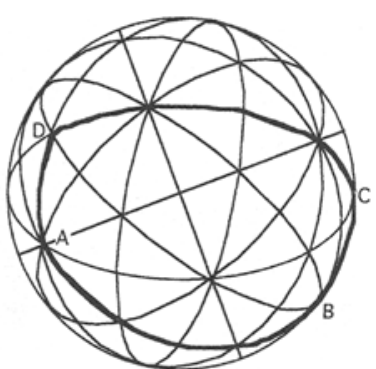

(c)

Fig. 8. (a) One face of $P \# 1=\{5,3\} \&\{3,5\}$, (b) $P \# 2=\left\{5, \frac{5}{2}\right\} \&\left\{\frac{5}{2}, 5\right\}$ and (c) $P \# 3=\left\{\frac{5}{2}, 3\right\} \&\left\{3, \frac{5}{2}\right\}$, all shown here in their projection $A B C D$ to the icosahedral Möbius net.

3.3. For the third method of construction we start with the three rhombic triacontahedra: the convex, the small stellated and the great stellated triancontahedra (see Plate 1 and Fig. 6.4C and D in [1], or pp. 121, 125, 126 in [3]). In each of these polyhedra every face can be paired with a congruent and parallel face, the two faces being related by reflection in the plane parallel to them and passing through the center of the polyhedron. Hence each such pair determines a "tunnel", the walls of which are four congruent rectangles; each rectangle has one edge in each of the two faces, and a pair of parallel edges joining the endpoints of these edges (see Figs. 9-11). Since each edge of the triacontahedron is in a plane of mirror symmetry of the polyhedron, the sixty rectangles that result from the fifteen pairs of parallel faces form an isohedral hexacontahedron of the kind considered. Specifically, $P \# 1$ is obtained from the great stellated triacontahedron, $P \# 2$ from the small stellated triacontahedron, and $P \# 3$ from the convex rhombic triacontahedron. Clearly, the tunnels described here coincide with the zones of the first construction; however, the logic of the two methods of construction is quite different.

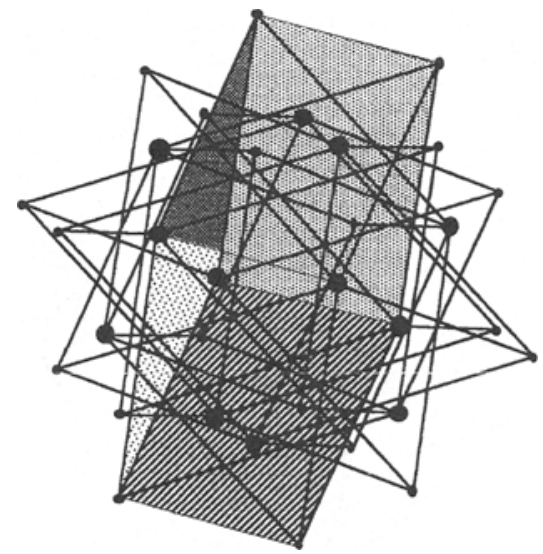

Fig. 9. Construction of $P \# 1$ from the great stellated triacontahedron. To avoid clutter, here and in Figs. 10 and 11 , only the four walls of the "tunnel" determined by one of the fifteen pairs of the parallel faces of the triacontahedron are shown. 


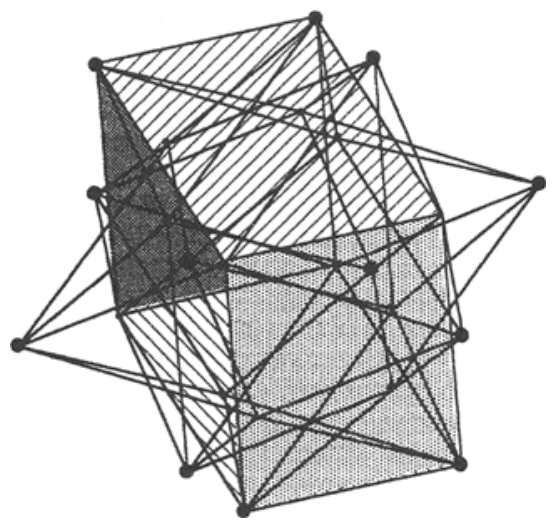

Fig. 10. Construction of $P \# 2$ from the small stellated triacontahedron.

For each of the three hexacontahedra, the sets of parallel edges of the rhombic triacontahedra used in the third construction determine twelve zones of five rectangles each. These are somewhat harder to visualize, but can easily be perceived using suitable models.

\section{Numerical Properties}

As we have seen, each of the three hexacontahedra has 60 faces and $60+60$ edges (of two lengths). $P \# 1$ and $P \# 3$ have $20+12$ vertices, respectively 6 -valent and 10 -valent. The twenty vertices are each surrounded by six rectangular faces, so that the vertex figure is an equilateral hexagon of density 2 with alternate angles of two sizes. The twelve vertices are each surrounded by ten rectangular faces, so that the vertex figure is an equilateral decagon of density 3 (for $P \# 1$ ) or 4 (for $P \# 3$ ).

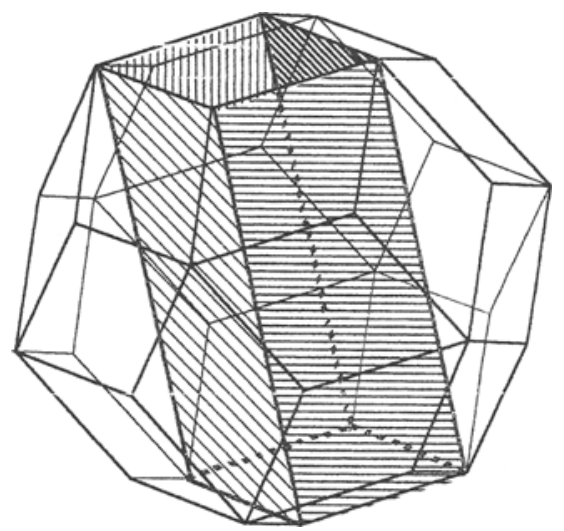

Fig. 11. Construction of $P \# 3$ from the convex thombic triacontahedron. 
On the other hand, $P \# 2$ has $12+12$ vertices, all 10 -valent; each vertex figure is an equilateral decagon, of density 3 for one kind of vertex and 4 for the other.

For each of the three hexacontahedra, the density is given by Cayley's formula [ 1 , equation 6.42 on p. 104]. Then for $P \# 1$ we find that the density is

$$
\frac{1}{2}(3 \cdot 12+2 \cdot 20-120+60)=8
$$

for $P \# 2$ it is

$$
\frac{1}{2}((3+4) \cdot 12-120+60)=12
$$

and for $P \# 3$ it is

$$
\frac{1}{2}(4 \cdot 12+2 \cdot 20-120+60)=14 \text {. }
$$

When regarded topologically as surfaces $[1$, p. 10], all three hexacontahedra are orientable. Polyhedra $P \# 1$ and $P \# 3$ each have Euler characteristic

$$
2-2 g=32-120+60=-28
$$

and thus genus $g=15$, while $P \# 2$ has characteristic

$$
2-2 g=24-120+60=-36
$$

and genus $g=19$.

\section{Metric Properties of the Hexacontahedra}

The metric properties of the hexacontahedra are most easily derived from the construction based on rhombic triacontahedra. For additional information about these polyhedra and their history see p. 115 in [1], and the references given there.

The vertices of the great stellated triacontahedron can be taken at the eight points $( \pm 1, \pm 1, \pm 1)$ with all choices of signs, and the twelve points $\left( \pm \tau^{-2}, \pm 1,0\right)$ with all choices of signs and cyclic permutations. From this it is easy to calculate that (in the notation of Fig. 8) the ratio $B C^{*} / A B$ of sides of the rectangular faces of $P \# 1$ is

$$
\frac{1}{2} \cdot 5^{1 / 4} \tau^{3 / 2}=1.538841768587626 \ldots
$$

Similarly, the twenty-four vertices of the small stellated triacontahedron have coordinates $( \pm \tau, \pm 1,0)$ and $\left( \pm 1, \pm \tau^{-1}, 0\right)$ with all choices of signs and cyclic permutations. Hence the ratio $B C^{*} / A B$ of the sides of the rectangular faces of the polyhedron $P \# 2$ is

$$
\frac{1}{2} \cdot 3^{1 / 2}=0.866025403784439 \ldots
$$

Finally, eight vertices of the convex rhombic triacontahedron are at the points $( \pm 1, \pm 1$, \pm 1 ) with all choices of signs, and the other twelve are at the points $\left( \pm \tau^{-1}, \pm \tau, 0\right)$ with all choices of signs and cyclic permutations. This shows that the ration $B C^{*} / A B$ of sides of the faces of $P \# 3$ is

$$
\frac{1}{2} \cdot 5^{1 / 4} \tau^{-3 / 2}=0.3632712640026805 \ldots
$$




\section{Comments}

As mentioned in [2], isohedra with non-square rectangles as faces do not seem to have been considered by other authors. It also seems that although Möbius nets have long been used in the study of uniform and other isogonal polyhedra, their applicability to investigations of isohedra seems not to have been noticed prior to [4].

The complete enumeration of rectangle-faced isohedra with all edges in mirrors of the icosahedral symmetry group can be based on the following simple observation (see [4]): If $Q$ is a spherically convex quadrangle, the cone generated by $Q$ with apex at the center of the sphere can be intersected by a plane in such a way that the intersection is a parallelogram; moreover, all such planes are parallel, and hence the parallelograms are mutually homothetic and the parallelogram is uniquely determined up to size. The proof of this assertion reduces at once to showing that for every point inside an angle (pointed cone) in the plane there is a unique chord that has the point as its midpoint. Clearly, the center of the parallelogram will be on the line of intersection of the two planes determined by the diagonals of the spherical quadrangle. The precise shape of the parallelogram can be easily determined, either trigonometrically or using numerical calculations.

With very little effort it is possible to verify that in the icosahedral Möbius net there are just forty-one different convex quadrangles. Precisely six of the resulting parallelograms are rectangles: the three shown in Fig. 8, and additional three which are obtained from them by taking the halves determined by midlines that are mirrors. These last three lead to polyhedra, the 120 faces of which are rectangles that are coplanar in pairs, and therefore are easily obtainable from our three hexacontahedra. The fact that they are isohedra with 120 faces is of some interest since it shows that polyhedra of this kind need not have triangular faces.

The arguments just made show that our enumeration of rectangle-faced isohedra with icosahedral symmetry is complete if we restrict attention to polyhedra of the KeplerPoinsot type. On the other hand, if faces that coincide as sets of points but are distinguished combinatorially (for example, by being "red" or "green", with only differentcolored faces sharing edges, or by some other means), then many other possibilities arise; these are not pursued here.

The rectangle-faced icositetrahedron $P_{1}$ described in [2] $]^{1}$ and constructed there by the analogue of the first method used here, can also be obtained by the other two approaches. The construction using Möbius nets appears in a more general context in [4] and yields both icositetrahedra $P_{1}$ and $P_{2}$ from [2]. On the other hand, the "tunnel" construction applied to the rhombic dodecahedron yields $P_{1}$. Here each rectangle joins an edge of the octahedron $\{3,4\}$ to a parallel edge of one of the two tetrahedra which constitute the stella octangula $\{4,3\}[2\{3,3\}]\{3,4\}$ (see p. 48 in [1]). So a suitable analogue for $P_{1}$ to the symbol $\{5,3\} \&\{3,5\}$ could be $2\{3,3\} \&\{3,4\}$.

\footnotetext{
1 The skeletal diagram of the icositetrahedron $P_{1}$ shown in the left part of Figure 2 of [2] was distorted during printing; it should have the same dimensions as the perspective view in the right part of this diagram. The labels (a) and (b) for the two parts were lost during the production, as were the ones in Figure 4 of [2].
} 


\section{References}

1. H. S. M. Coxeter, Regular Polytopes, third edition. Dover, New York, 1973.

2. H. S. M. Coxeter and B. Grünbaum, Face-transitive polyhedra with rectangular faces. C. R. Math. Rep. Acad. Sci. Canada 20 (1998), 16-21.

3. H. M. Cundy and A. P. Rollett, Mathematical Models, second edition. Clarendon Press, Oxford, 1961.

4. B. Grünbaum, Parallelogram-faced isohedra with edges in mirror-planes. Discrete Math. 221 (2000), 93100.

Received March 18, 1999, and in revised form July 8, 2000. Online publication January 17, 2001. 